\title{
Arrhythmias in the Adult with Congenital Heart Disease
}

Author: Seshadri Balaii

Department of Pediatrics, Division of Pediatric Cardiology, Oregon Health \& Science University

Address for correspondence:

Associate Professor, Seshadri Balaji

707, SW Gaines Road (Mail code CDRC-P)

Portland, OR 97239

USA

Email:

balaji@@ohsu.edu

\section{ABSTRACT Arrhythmias are an important problem} in adults with congenital heart disease, whether previously operated upon or not. This article discusses the incidence, pathogenesis, diagnosis and management of arrhythmias in the adult congenital patient. Arrhythmias can be the cause or the effect of hemodynamic deterioration. Complex patients, such as those with transposition of the great arteries, tetralogy of Fallot and single ventricle problems are highly likely to have major arrhythmias during adult life. Early recognition and appropriate management can improve the patient's life and can even prevent early death. Pacemaker placement should be considered for bradycardia. Tachyarrhythmia patients may need a combination of drug therapy, catheter ablation, pacemaker for antitachycardia pacing, or reoperation for residual structural issues combined with arrhythmia surgery. Sudden death is another major problem and risk stratification strategies will be discussed.Appropriate and early use of implantable defibrillators can also save lives.

\section{INTRODUCTION}

Arrhythmias are an important problem in adults with congenital heart disease $(\mathrm{CHD})$ and cause morbidity and mortality whether the $\mathrm{CHD}$ was previously repaired or not. Arrhythmias may be the result of natural history of the underlying congenital cardiac abnormality (e.g. heart block in congenitally corrected transposition of the great arteries
(CCTGA), the (longstanding) hemodynamic issues caused by the heart defect, and/or the result of prior surgery. In general, atrial arrhythmias are seen in patients who have atrial defects (such as atrial septal defect (ASD)) or who have undergone extensive atrial surgery (e.g. Mustard's operation for transposition of the great arteries (TGA) or the Fontan operation for single ventricle) and ventricular arrhythmias occur in patients who have undergone ventricular surgery (e.g. tetralogy of Fallot (TOF) or double outlet right ventricle). However, there are many exceptions. Atrial arrhythmias, for instance, may be the result of longstanding atrial hypertrophy secondary to a noncompliant ventricle and are often seen in adults with repaired TOF.

It is also important to keep in mind the close interaction between arrhythmia and hemodynamic problems. Indeed it may be hard to say which is the cause and which the effect in a given patient. Longstanding hemodynamic problems can lead to arrhythmias which, in turn, lead to further hemodynamic deterioration. Similarly, chronic bradycardia or junctional rhythm with atrio-ventricular (AV) dissociation can be the cause of cardiac failure.

\section{PREVALENCE}

The remarkable success of cardiac surgery for CHD has now created a large pool of adults with CHD in developed countries. Indeed, it is now estimated that in countries such as the USA, for the first time in history there are more adults than children with $\mathrm{CHD}^{(1)}$ In these patients, there are specific arrhythmias that are seen (such as incisional atria reentrant tachycardia (IART)), which impose a severe burden of care. In the developing world, it is mostly patients with the simpler heart defects (such as ASD, ventricular septal defect (VSD),TOF, etc.) who survive to adulthood, but the natural history of these defects also makes them prone to arrhythmias (atrial fibrillation (AF) for instance in the unrepaired ASD patient).

\section{BRADYARRHYTHMIAS}

In general, bradyarrhythmias are rare in the unoperated patient and the commonest cause of significant bradycardia is surgical trauma to either the sinus or AV node. 
In the rare patient with heterotaxy syndrome due to left atrial isomerism (usually associated with polysplenia) there may be a developmental absence or maldevelopment of the sinus node with bradycardia. ${ }^{(2)}$

In patients with CCTGA (or single ventricle with CCTGA-like ventricular relationship) there is a natural tendency towards $\mathrm{AV}$ block with bradycardia. This is due to the fact that these patients have an AV node that is anteriorly displaced. These patients also are more vulnerable to developing heart block during surgery for VSD (a common associated condition). ${ }^{(3)}$

Sinus node dysfunction is a common problem late after surgery in the region of the node or its arterial supply. The commonest patients with this problem are those who have had an atrial repair (Mustard's or Senning's operation) for TGA, and Fontan operation for functional single ventricles. Both of these operations involve much atrial dissection and suture lines. Sinus node damage used to be a problem in patients undergoing ASD repair, but this has largely been resolved with better knowledge of the anatomy of the sinus node and its arterial supply. ${ }^{(4)}$

AV node damage is usually secondary to operations such as VSD, TOF, $A V$ canal and CCTGA with VSD repair. While most of these occur immediately after surgery in childhood, there is a small incidence of late appearing AV block. ${ }^{(5)}$ Management of bradyarrhythmias is by pacemaker implantation.

\section{PACEMAKER IMPLANTATION}

Endocardial pacemaker (or implantable cardioverter defibrillator (ICD)) implantation can be far from routine in CHD patients. It is important to perform angiography of the veins that the operator hopes to use to make sure there are no congenital venous abnormalities (such as left superior vena cava draining to the coronary sinus) or acquired venous abnormalities such as stenoses or occlusion from prior cannulation for surgery, peri-operative care or cardiac catheterization. For instance, patients who have had Mustard's operation for TGA may have stenoses in their baffles that redirect systemic venous blood. ${ }^{(6)}$

A thorough knowledge of the patient's anatomy and prior surgery is important. For instance, during the Fontan operation, the right atrium (or the great veins) is directly connected to the pulmonary arteries and thus venous access to the ventricles is lost. Residual intracardiac right to left shunts may pose a problem since pacemaker patients may develop thrombi around the leads with potential for systemic embolization.
Knowledge of such aspects may cause the team to opt for an epicardial approach in some patients.

\section{TACHYARRHYTHMIAS}

Wolff Parkinson White syndrome,Accessory Pathways and AV nodal reentrant tachycardia

Patients with Ebstein's anomaly of the tricuspid valve have a significant incidence of accessory pathways with a higher likelihood of multiple accessory pathways as well. (7) They are prone to AV reentrant tachycardia. As seen in patients with Wolff Parkinson White syndrome (WPW) without CHD, they also have a small risk of sudden death. While catheter ablation is usually curative, the ablation procedures are more difficult due to the complex anatomy of the tricuspid valve annulus, with lower acute success and a higher chance of pathway recurrence. ${ }^{(8)}$

Although not technically an accessory pathway, some rare patients with ambiguous atrial arrangement (heterotaxy syndrome) can have two AV nodes, one anterior and the other posterior, with a sling of conduction tissue (the Monckeburg sling) connecting the two, with the possibility of a rare form of $A V$ nodal reentrant tachycardia where the circuit goes from one AV node to the other and then turns around in the atrium. $^{(9)}$

Even otherwise, patients with CHD may develop dual AV nodal pathways and go on to have AVNRT.

The main treatment options are medical therapy with drugs or catheter ablation.

\section{INCISIONAL ATRIAL REENTRANT TACHYCARDIA (IART) AND ATRIAL FLUTTER (AFL)}

Atrial flutter (AFL) is an important problem in adults with unrepaired ASDs. Repair in childhood appears to prevent the development of AFL. Later repair in adulthood seems to prevent arrhythmias but does not help if the arrhythmias have already started to occur. ${ }^{(10,11)}$

IART goes by many names such as atypical AFL, scar-based AFL or sometimes just plain AFL. However, it is quite different from AFL in patients without CHD. This is not to say that AFL of the usual variety does not happen in CHD patients. Atrial stretch due to longstanding unrepaired CHD can lead to AFL that rotates around the cavo-tricuspid isthmus (as it does in normal hearts and those with unrepaired ASD). 


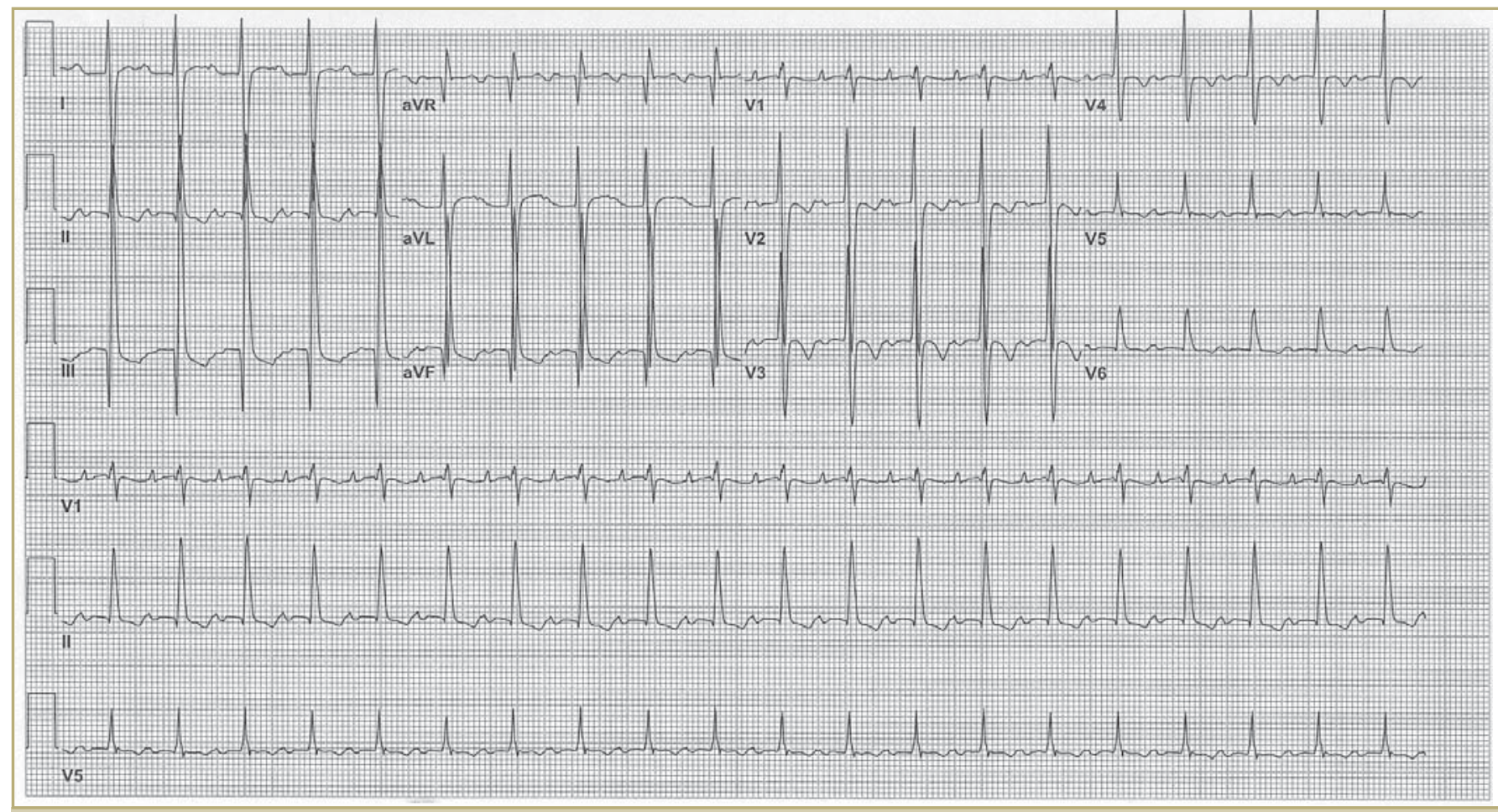

FIGURE 1: Slow incisional atrial reentrant tachycardia (IART) with 1:1 atrioventricular conduction in an adult Fontan patient. The rhythm can easily be mistaken for sinus.

Also, many patients with IART have rhythms that utilize the cavotricuspid isthmus. ${ }^{(12)}$ However, even in these two categories of patients, the rhythm tends to be slower and may not show the typical sawtoothed $\mathrm{p}$-waves associated with usual atrial flutter because of the underlying CHD (Figure I).

IART is a macro-rentrant atrial tachycardia where the rhythm rotates around one or more scars in the atrium. It predominantly tends to be in the right (systemic venous) atrium, and the atrial rate is slower than typical AFL. While any atrial scar can lead to IART, the incidence seems to increase with complexity of surgery and other residual problems that lead to atrial hypertrophy or stretch. Thus, it can occur after a "simple" ASD operation, but tends to be more common after atrial repair (Mustard's or Senning's type) for TGA. The most commonly affected patient is one who has had a Fontan operation (or one of its numerous modifications). ${ }^{(12)}$

IART tends to be slower in rate, which is a disadvantage since it predisposes to $1: 1$ conduction to the ventricle, making the patient more symptomatic. Furthermore, the atrial scars can bestow unusual shapes to the $p$-wave (or even rarely the absence of $p$ waves or lowamplitude, fragmented $p$ waves). P-waves can be hard to see and the rhythm may be mistaken for something else, such as sinus tachycardia.
The clue, however, is in the patient's symptoms, and the fact that most of these patients have underlying sinus node dysfunction. It is not unusual to see so-called "tachycardias" with rates as low as 100-150 The diagnosis can be made manifest with administration of adenosine, which usually unmasks $p$ waves hiding under the QRS complexes. Also, it is not unusual for a single patient to have multiple IART circuits.

Acute treatment of IART to convert the rhythm to sinus consists of: electrical cardioversion, chemical cardioversion with drugs such as ibutilide, amiodarone, etc. and overdrive pacing, either via a transesophageal or intracardiac atrial electrode, or via a pre-existing pacemaker.

Chronic treatment is directed mainly towards rhythm control (rather than rate control, which is sometimes used as a last resort) and anticoagulation to prevent thromboembolic complications. Rhythm control may be achieved using one of four options: drug therapy, catheter ablation, atrial anti-tachycardia pacing and surgical ablation.

Drug therapy often involves the use of class 3 (sotalol, amiodarone, dofetilide) or IC (flecainide, propafenone) antiarrhythmics. These potent drugs come with the risk of lethal proarrhythmia and need to be initiated in hospital. Furthermore, they can exacerbate the preexistent 
tendency to bradyarrhythmia and push the patient towards need for a pacemaker. Chronic use of amiodarone can also lead to multisystem problems such as hepatic failure, thyroid abnormalities, pulmonary disease and optic neuropathy.

Catheter ablation is now becoming the rage for IART since it offers the hope of a cure without too much risk. However, the anatomical and electrophysiological complexity of these patients leads to a lower immediate success rate, and worse, a significant incidence of recurrence. The procedures can be long and complex and need specialized laboratories and specialized personnel to be successful. Detailed mapping of the tachycardia circuits is important.(12) The advent of 3-dimensional electroanatomical mapping systems such as the CARTO (Biosense Webster, diamond bar, CA, USA) and Endocardial Solutions (St Jude Medical, St Paul, MN, USA) systems have improved our understanding of the rhythm circuits and the exact anatomy of the suspect atrium. Despite the advent of newer catheter technology (large tips, cooled tips, etc.) one big hurdle is the thickness of the atrial wall, which may preclude successful ablation despite successful mapping and understanding of the rhythm. ${ }^{(13)}$

Antitachycardia pacing is an option in some patients. While it does not prevent the arrhythmia, it can successfully convert $~ 50 \%$ of arrhythmia episodes with decrease in emergency room visits and hospitalizations. ${ }^{(14)}$ Currently available options include the Enrhythm pacemaker (Medtronic). The additional advantage of these pacemakers is the prevention of bradycardia.

Surgical ablation is a last resort option in some patients. The Fontan patient is the one that most needs this option. Dr Mavroudis and colleagues from Children's Memorial Hospital in Chicago have demonstrated the benefits of converting patients from the older style Fontan operation (atrio-pulmonary connection) to the newer style extra-cardiac connection, combined with creation of a right atrial Maze, and implantation of a pacemaker. However, this approach is not without significant morbidity and some mortality. ${ }^{(15)}$

\section{ATRIAL FIBRILLATION}

Atrial fibrillation (AF) may be the result of chronic left atrial pressure or volume stress as seen in patients with chronic left heart lesions (aortic, mitral disease or unrepaired single ventricle, etc.) or the end stage of chronic uncontrolled IART. It tends to differ from AF in non-CHD patients as seen by large fibrillatory waves in the ECG (Figure 2). Some patients may present with an intermediary stage between AFL and AF with periods that tend to look more organized than others. Nevertheless,

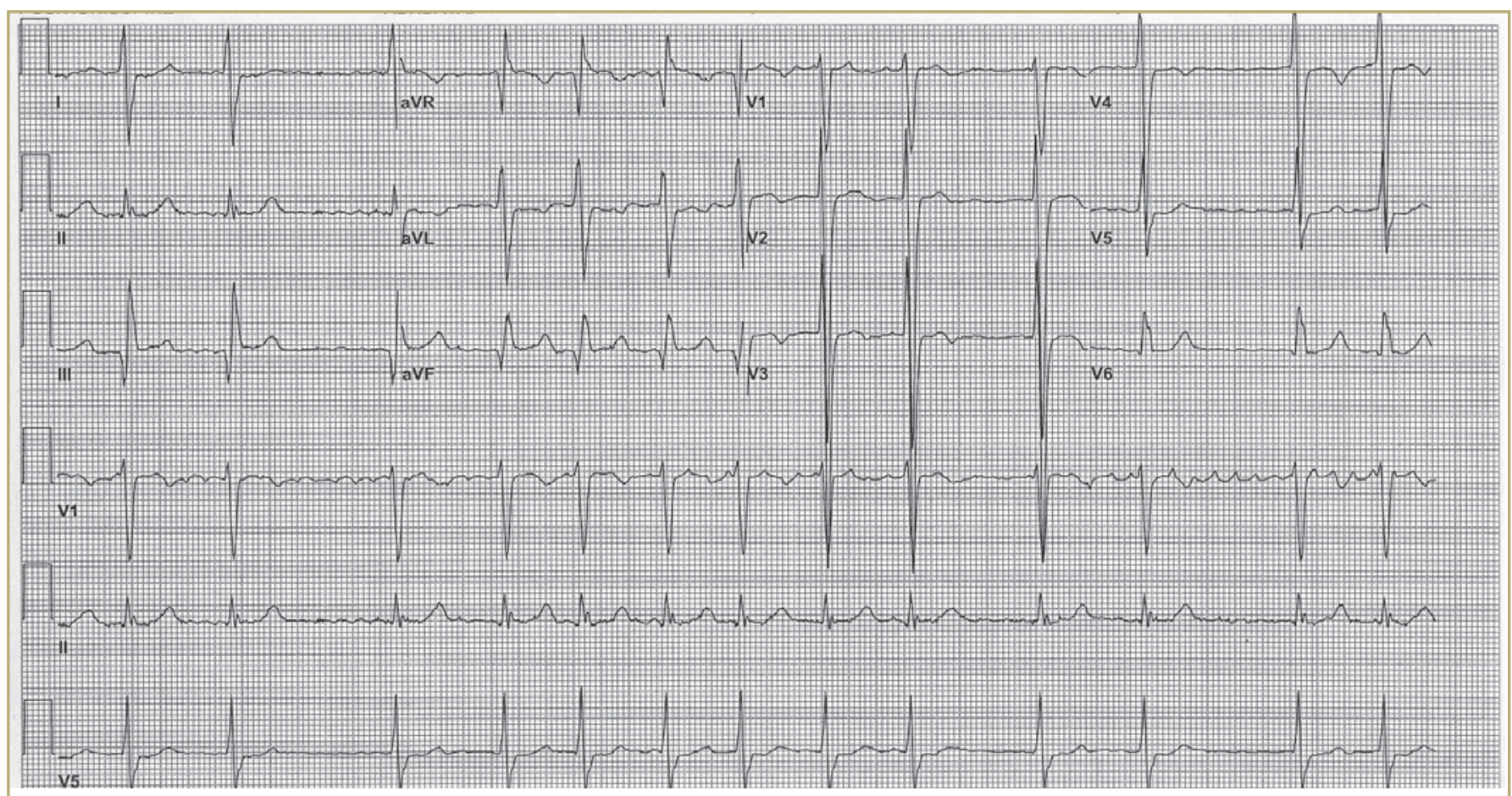

FIGURE 2: Atrial fibrillation in an adult Fontan patient. Note the coarse fibrillatory waves in the V1 rhythm strip. 
ARRHYTHMIAS

IN CONGENITAL

HEART DISEASE

from the electrophysiologic point of view, the atriums are in electrical chaos and the patient has a high risk of thromboembolic problems.

Apart from anticoagulation, therapy tends to be limited to two options: drugs versus surgical Maze operation. Catheter ablation has not so far been described. While pacing has been suggested to prevent $A F$, this is not proven in the CHD patient.

\section{VENTRICULAR TACHYCARDIA}

$V T$ is mainly seen in patients who have had surgical scars in the ventricle or those who have had longstanding ventricular hypertrophy or dilatation or both. The combination of scar-hypertrophy-dilation seems to be the most important. Scar alone may not be as arrhythmogenic as seen by the relative paucity of VT in patients who have only had a VSD closure. The combination of dilatation-hypertrophy may also be arrhythmogenic as seen in patients who have had longstanding aortic or pulmonary valve disease. In CCTGA or after atrial repair ofTGA, the systemic RV is enlarged and hypertrophied and can be a source of $V T$. This is the likely mechanism in unoperated patients with ventricular pressure/volume overload as well although, in all these groups, microscarring and fibrosis has been demonstrated. ${ }^{(16)}$

The literature on VT in CHD is dominated by TOF patients. This is probably because this is a relatively homogenous defect which has been repairable since the early days of CHD surgery. These patients often have residual pulmonary valve problems such as stenosis or, more commonly, significant regurgitation. Of these, the severity and duration of regurgitation with consequent $\mathrm{RV}$ dilation (demonstrable on $\mathrm{X}$-ray, echo, $M R I$ and even the ECG with the QRS duration prolongation) seems to be the most important offender. Other lesions such as post-Rastelli-type repair of double outlet $\mathrm{RV}$ are probably just as likely to get $\mathrm{VT}$.

$\mathrm{VT}$ can degenerate to VF and sudden death as seen by the risk of SD in patients with VT or the same substrates that cause VT.

Management of $\mathrm{VT}$ consists acutely of electrical (DC current) cardioversion or pharmacologic conversion with drugs like i.v. lidocaine, procainamide or amiodarone. Chronic management with drugs may be possible in the rare patient with monomorphic $V T$ who "tolerates" the VT, but usually requires the use of class 3 drugs (sotalol or amiodarone) or IC drugs (flecainide, propafenone). In developed countries, the occurrence of $\mathrm{VT}$ is usually taken to indicate a high risk of SD with need for ICD placement unless the VT is mappable and ablatable either by catheter (a rare occurrence as seen by the paucity of reports in the literature) or by surgical means. Antitachycardia overdrive pacing out of $\mathrm{VT}$ may be possible using ICDs. These options are applicable to the patient with monomorphic VT. Polymorphic VT seems to affect patients with significant ventricular dysfunction and may be a more important precursor to sudden death and should be considered an indication for an ICD.

\section{SUDDEN DEATH}

Oechslin et al. showed that sudden death is one of the most common modes of late death in patients with CHD. Indeed, in some conditions, it is the commonest mode of death. Apart from the very simple defects (such as repaired ASD), there is an incidence of SD in almost every defect. ${ }^{(17)}$

Silka et al. reported a population-based study of 25-year follow-up after surgery for CHD. The incidence of late $\mathrm{SD}$ in CHD patients is $\sim 0.9 / 1,000$ patient-years. This compares unfavorably to the general risk of I per 25000 to 100000 patient-years in persons $<20$ years. They found that 4 diagnostic categories accounted for $90 \%$ of late sudden deaths: tetralogy of Fallot, D-transposition of the great arteries, coarctation of the aorta and aortic stenosis. The SD rate for patients with these defects was 2.2/1000 patient-years as compared to 0.14/1000 for patients with left to right shunt lesions or pulmonary stenosis. Eisenmenger's syndrome is also an important sub-group with risk of SD. ${ }^{(18)}$

The cause of SD is thought to be VT/NF in most cases. However, the possibility that AFL/AF/IART immediately precedes SD is not yet disproven. The advent of implantable defibrillators has changed the outlook for patients felt to be at risk of SD. However, ICDs come with enough disadvantages to make them impractial for every patient with a serious CHD. These disadvantages include the need for surgery (which sometimes has to quite creative, as noted in the section on pacing), the need for multiple surgeries, inappropriate shocks (which are more common in younger patients), and cost. These factors make it important to determine who exactly is at risk for SD.

As far as risk stratification is concerned, the literature on SD is also heavily tilted towards TOF. Some of the factors that have been shown to influence risk of SD are: older age at surgery, wide QRS (>180ms) on the ECG, frequent and complex ventricular ectopics, RV dilation, and positive electrophysiologic studies.(19) Despite much work, risk stratification remains imprecise. Apart from the symptomatic patient with syncope, dizziness, and rarely, near-miss SD, who shows some of the high risk features above, it is hard to decide which patient will 
benefit from an ICD. A positive EP study with induction of sustainedVT or VF may help but non-inducibility does not rule out risk of SD. (20-22) Risk stratification in the asymptomatic patient is even more difficult. The role of primary prevention of SD using an ICD remains open to study.

SD in patients after atrial repair of TGA has been shown to be associated with prior episodes of IART and with symptoms of heart failure. ${ }^{(23)}$ This reinforces the notion of a detrimental vicious circle with heart failure and atrial arrhythmias eventually leading to ventricular failure and SD. However, the possibility that IART with acute deterioration of ventricular function and development of VF is the cause of SD cannot be disproved Anecdotally, naany episodes of SD seem to happen during exercise or exertion. Therefore, activity recommendations with restriction from strenuous sports or exercise are important in patients thought to be at risk for $\mathrm{SD}$

\section{CONCLUSIONS}

Arrhythmias are a big challenge in ACHD patients. The complexity of the underlying anatomy, poor response to the usual therapies and significant hemodynamic deterioration secondary to arrhythmias, make it imperative that clinicians stay pro-active and vigilant in caring for these patients.

Much knowledge has been gained in the past two decades and significant advances have been made, including the advent of catheter ablation, ICDs, and newer drugs (e.g. amiodarone). Despite this, much work remains. There is hope that the trend towards earlier repair of $\mathrm{CHD}$, more physiologic repair (e.g. the arterial switch compared to the Mustard's/Senning's operation) and newer surgical modifications (e.g. avoiding transannular patching in tetralogy, the extracardiac conduit Fontan, etc.) may prevent or, at least, retard the development of arrhythmias in the future. Further innovations such as newer drugs and improved ablation techniques will also play a role in improving the outlook for these patients. Indeed, we must remember that in many patients, arrhythmias are the result of success, not failure.

\section{REFERENCES:}

I. Warnes CA, Liberthson R, Danielson GK, et al. "Task force I: the changing profile of congenital heart disease in adult life." J Am Coll Cardiol. 200 I; 37(5): I I 70-5.

2. Wu MH, Wang JK, Lin LP. et al. "Cardiac rhythm disturbances in patients with left atrial isomerism." Pacing Clin Electrophysiol. 200 I; 24(1 I): I631-8.

3. Warnes CA. "Transposition of the great arteries." Circulation. 2006; I I 4(24): 2699-709.

4. Bink-Boelkens MT, Meuzelaar KJ, Eygelaar A. Arrhythmias after repair of secundum atrial septal defect: the influence of surgical modification. Am Heart J. 1988 Mar; II5(3): 629-33.

5. Bonatti V, Agnetti A, Squarcia U. "Early and late postoperative complete heart block in pediatric patients submitted to open-heart surgery for congenital heart disease." Pediatr Med Chir. 1998; 20(3): 181-6.

6. Gewellig M, Cullen S, Mertens B, et al. Risk factors for arrhythmia and death after Mustard operation for simple transposition of the great arteries. Circulation 1991; 84 (suppl): |||- | 87|||- 192.

7. Celermajer D, Bull C, Till J, et al. Ebstein's anomaly: Presentation and outcome from fetus to adult. J Am Coll Cardiol 1994; 23:170- 176.

8. Reich JD, Auld D, Hulse E, et al. "The Pediatric Radiofrequency Ablation Registry's experience with Ebstein's anomaly. Pediatric Electrophysiology Society." J Cardiovasc Electrophysiol. 1998; 9(12): 1370-7.

9. Epstein MR, Saul JP, Weindling SN, et al. "Atrioventricular reciprocating tachycardia involving twin atrioventricular nodes in patients with complex congenital heart disease.' J Cardiovasc Electrophysiol. 200 I; 12(6): 67I-9.

10. Gatzoulis M, Freeman M, Siu S, et al. Atrial arrhythmia after surgical closure of atrial septal defects in adults. N Engl J Med 1999; 340:839-846.

I I. Swan L and Gatzoulis MA. "Closure of atrial septal defects: is the debate over?" Eur Heart J. 2003; 24(2): 130-2.

12. Triedman J, Saul J, Weindling S, et al. Radiofrequency ablation of intra-atrial reentrant tachycardia after surgical palliation of congenital heart disease. Circulation 1995; 91: 707-7|4.

13. McGreevy KS, Hummel JP, Jiangang Z, et al. "Comparison of a saline irrigated cooled-tip catheter to large electrode catheters with single and multiple temperature sensors for creation of large radiofrequency lesions." J Interv Card Electrophysiol. 2005; 14(3): I39-45.

14. Stephenson EA, Casavant D, Tuzi J, et al. Efficacy of atrial antitachycardia pacing using Medtronic AT500 pacemaker in patients with congenital heart disease. Am J Cardiol. 92, 87I-876 (2003)

15. Mavroudis C, Backer CL, Deal BJ, et al. "Evolving anatomic and electrophysiologic considerations associated with Fontan conversion." Semin Thorac Cardiovasc Surg Pediatr Card Surg Annu. 2007; I 36-45.

16. Chowdhury UK, Sathia S, Ray R, et al. "Histopathology of the right ventricular outflow tract and its relationship to clinical outcomes and arrhythmias in patients with tetralogy of Fallot." J Thorac Cardiovasc Surg. 2006; I 32(2): 270-7.

17. Oechslin EN, Harrison DA, Connelly MS, et al. Mode of death in adults with congenital heart disease. Am J Cardiol. 2000 Nov 15; 86( I0): I I I -6.

18. Silka M, Hardy B, Menashe V, et al. A population-based prospective evaluation of risk of sudden cardiac death after operation for common congenital heart defects. J Am Coll Cardiol 1998; 32:245-25I.

19. Gatzoulis M, Balaji S, Webber S, et al. Risk factors for arrhythmia and sudden death in repaired tetralogy of Fallot: A multi-centre study. Lancet 2000; 356:975-98I.

20. Balaji S, Lau YR, Case CL, Gillette PC. QRS prolongation is associated with inducible ventricular tachycardia after repair of tetralogy of Fallot. American Journal of Cardiology, 1997:80: 160-163

21. Alexander ME, Walsh EP, Saul JP, et al. "Value of programmed ventricular stimulation in patients with congenital heart disease." J Cardiovasc Electrophysiol. 1999; 10(8): 1033-44.

22. Khairy P, Landzberg MJ, Gatzoulis MA, et al. "Value of programmed ventricular stimulation after tetralogy of Fallot repair: a multicenter study." Circulation. 2004; 109( I 6): 1994-2000.

23. Kammeraad JAE, Van Deurzen CHM, Sreeram N, et al. Predictors of sudden death after Mustard or Senning repair for transposition of the great arteries. J Am Coll Cardiol.2004;44: 1095-1 102. 